\title{
Accelerating Airy Beams Generated by Ultrafast Laser Induced Space-Variant Nanostructures in Glass
}

\author{
Mindaugas Gecevičius, Martynas Beresna and Peter G. Kazansky \\ Optoelectronics Research Centre, University of Southampton, SO17 1BJ, United Kingdom \\ mg2e10@orc.soton.ac.uk
}

\begin{abstract}
We demonstrate new technique to generate accelerating Airy beam with femtosecond laser imprinted space variant birefringence produced by self-assembled nanostructures in fused silica. The technique enables dual Airy beam generation.
\end{abstract}

OCIS codes (140.3390) Laser materials processing; (260.7120) Ultrafast phenomena; (260.1440) Birefringence

The first experimental demonstration of finite-energy optical Airy beams was demonstrated by Siviloglou et al. [1]. The envelope of such beams is described by an Airy function. The most interesting attribute of Airy beams is their ability to freely accelerate in the transverse direction during propagation even in the absence of any external potential. These beams were shown to be useful for optical micro-manipulation of small particles and the generation of curved plasma channels. Also they are unique since they are the only nonspreading solutions in one dimension [1].

Angular Fourier spectrum of an exponentially truncated Airy function is a Gaussian beam modulated with a cubic phase. In this context, in order to generate Airy beams, the cubic phase has to be imprinted on a Gaussian beam and Fourier transform has to be applied by using a lens. Usually, the cubic phase modulation in an optical beam is created by means of liquid crystal spatial light modulators (SLMs). However, this method requires complicated and expensive setup, which additionally puts a limitation on the input laser power. We present a simple and effective way of generating Airy beams by exploiting femtosecond laser induced nanogratings in fused silica glass. The nanogratings are responsible for the laser induced birefringence and can be employed for controlling both the spatial distribution of the light polarization and the phase in a laser beam [2]. If the laser induced retardance has a value of half wave, by controlling orientation of slow axis angle in the structure, we can manipulate polarization direction of incident linearly polarized light [3]. If incident light is circularly polarized, the phase of the transmitted light will depend on the slow axis angle [4].
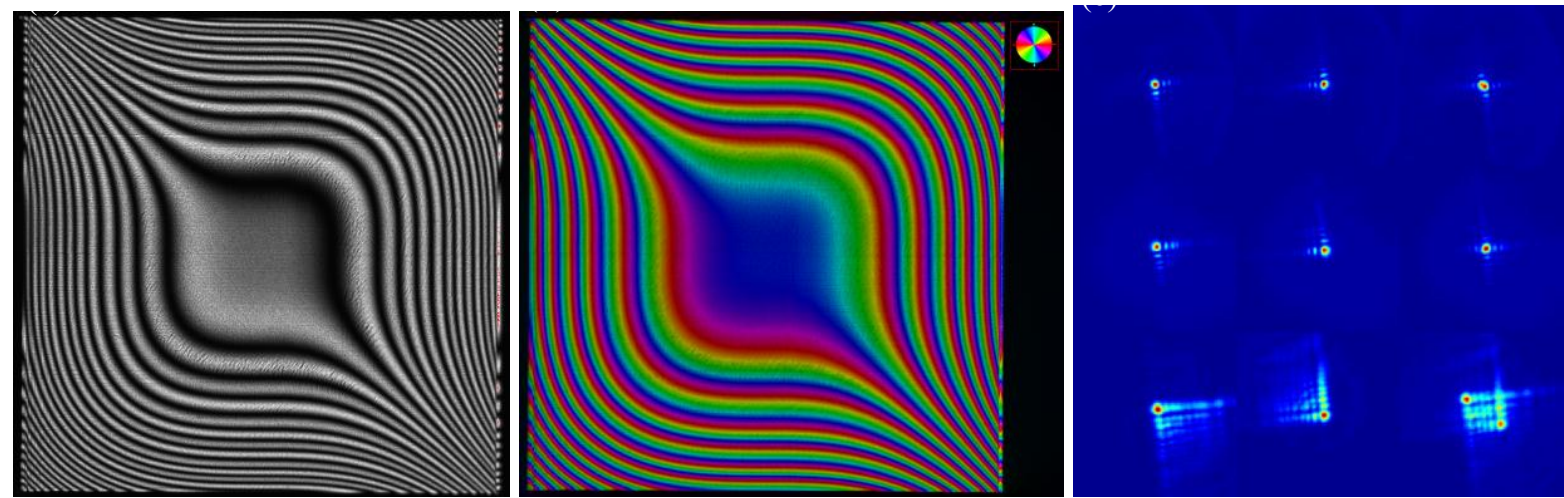

Fig. 1. Microscopic image of structure between cross-polarizers (a) and quantitative birefringence microscopy image (b), where pseudocolor represents slow axis angle. Structure size is $2 \times 2 \mathrm{~mm}$. Profiles of generated Airy beam in different distances (c). In the first and second column Airy beams are generated from the incident beams of left- and right-handed circular polarization while the third column shows a dual Airy beam which was generated from linear polarization.

To write the Airy beam converter, regeneratively amplified, mode-locked $\mathrm{Yb}: \mathrm{KGW}$ based femtosecond laser system (Pharos, Light Conversion Ltd.) operating at $1030 \mathrm{~nm}$ and delivering pulses of $270 \mathrm{fs}$ (stretched to $800 \mathrm{fs}$ ) at $200 \mathrm{kHz}$ repetition rate was utilized. The pulse energy was set to $0.75 \mu \mathrm{J}$. The light beam was focused via a $\times 10$ $(0.16 \mathrm{NA})$ aspheric lens into a fused silica plate $300 \mu \mathrm{m}$ below the surface. The control of the incident beam polarization azimuth was performed with an achromatic half-wave plate mounted into a motorized rotation stage. The sample was placed onto a three-dimensional translation stage. Structure of $2 \times 2 \mathrm{~mm}$ size was fabricated at 2 $\mathrm{mm} / \mathrm{s}$ speed. Nanogratings written under these conditions induce retardance of $\sim 266 \mathrm{~nm}$, which corresponds to half wave for $532 \mathrm{~nm}$ wavelength. 
In order to genearate Airy beams, the CW laser light from an $\mathrm{Ar}^{+}$-ion laser was launched through the fabricated structure and a converging lens (Fig. 1. (c)). Phase delay in such structure is sensitive to the handedness of circular polarization which means that acceleration direction of Airy beam depends on the incident polarization handedness (the first and the second column in Fig. 1 (c)). As linear polarization can be described as a superposition of two circular polarizations, the superposition of two Airy beams can easily be generated (the third column in Fig. 1 (c)). Until now a dual Airy beam is described only theoretically [5] and here we present its first experimental observation.

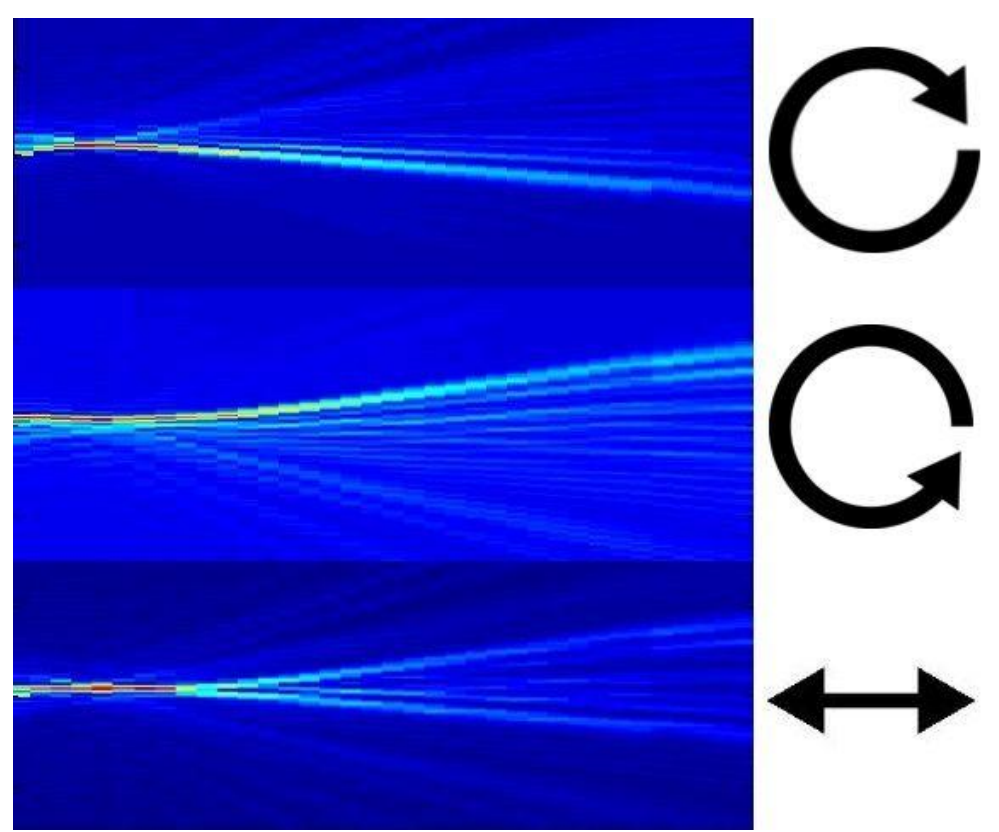

Fig. 2. Propagation of 1D Airy beam. Arrow indicates the polarization of incident Gaussian beam.

Also structure for 1D Airy beam generation was produced. Propagation of generated 1D Airy beams is demonstrated in Fig. 2. For different handedness of circular polarization generated Airy beam bends to different direction. If incident Gaussian beam is linearly polarized - 1D dual Airy beam is generated.

In summary, exploiting the ability of femtosecond lasers to imprint birefringent modification inside fused silica glass, we fabricated Airy beam converter working at $532 \mathrm{~nm}$ wavelength and demonstrated generation of 1D and 2D Airy beams. We have shown that this technique allows for the generation of a dual Airy beams.

\section{References}

[1] G. Siviloglou, J. Broky, a Dogariu, and D. Christodoulides, "Observation of Accelerating Airy Beams," Physical Review Letters 99, 23-26 (2007).

[2] E. Bricchi, B. G. Klappauf, and P. G. Kazansky, "Form birefringence and negative index change created by femtosecond direct writing in transparent materials," Optics letters 29, 119-21 (2004).

[3] M. Beresna, M. Gecevičius, P. G. Kazansky, and T. Gertus, "Radially polarized optical vortex converter created by femtosecond laser nanostructuring of glass," Applied Physics Letters 98, 201101 (2011).

[4] M. Beresna and P. G. Kazansky, "Polarization diffraction grating produced by femtosecond laser nanostructuring in glass," Optics letters 35, 1662-4 (2010).

[5] C.-Y. Hwang, D. Choi, K.-Y. Kim, and B. Lee, "Dual Airy beam," Optics express 18, 23504-16 (2010). 